\title{
El aporte educativo de las TIC a la construcción de la paz territorial en Colombia
}

\author{
José Javier Capera Figueroa \\ caperafigueroa@gmail.com \\ Instituto de Investigaciones Dr. José María Luis Mora
}

\section{Resumen}

La violencia en Colombia es un fenómeno estructural que ha marcado la vida de cualquier ciudadano por más de medio siglo, dicho panorama se ha configurado como una problemática política, económica, social y cultural que permite la constitución de prácticas desde abajo en función de superar las secuelas del conflicto armado en el país. Por ello, la finalidad del siguiente artículo de investigación consiste en señalar la importancia del componente educativo - pedagógico de las TIC, al ser un instrumento de formación de sujetos críticos, autocríticos, éticos, políticos y con una praxis orientada a construir experiencias de paz por medio de la difusión, la divulgación, la elaboración y la creación de espacios, relaciones y formas virtuales de comunicación del sujeto en comunidad. La metodología que se aplicó fue: el análisis crítico documental, la sistematización de fuentes primarias y secundarias, la revisión y reflexión teórico- analítica sobre las TIC, la violencia y la construcción de paz desde abajo a través de las herramientas virtuales en espacios locales y/o comunitarios.

\section{Palabras Clave}

TIC, Posconflicto, Violencia, Construcción de Paz, Educación.

\footnotetext{
Recibido 19/10/2019 - 12/11/2019.
} 


\section{Introducción}

Las sociedades del conocimiento o sociedades en red en las últimas décadas, se han convertido en el referente de análisis que permite conocer los avances, retrocesos, dinámicas y contradicciones de los distintos contextos en donde se constituyen procesos de comunicación por medio de la red. Así Manuel Castells ha generado una serie de elementos teórico-conceptuales para lograr tener un acercamiento sobre la constitución de la información, la comunicación y la cultura en el contexto individual y colectivo del sujeto. La necesidad de reconocer lo que representa la sociedad del conocimiento en pleno siglo XXI, implica todo un debate sobre la constitución del sujeto y la expansión del saber por medio de las prácticas de los actores en sus respectivos contextos. Por ello, las transformaciones en la técnica, la tecnología y la comunicación han dado un giro radical sobre la interacción individual y colectiva.

El conocimiento puede ser vinculado como una forma de poder en esta época, debido a su capacidad de difusión, relación y vinculación con la praxis del sujeto. Dicha situación, según Castells (2010) representa una oportunidad para generar alternativas de información, desinformación y transparencia sobre lo que se transmite y los grupos sociales reciben y reproducen en sus contextos locales, nacionales e internacionales.

La revolución tecnológica ha sido una etapa fundamental para la ciencia y el fortalecimiento del conocimiento, aunque en ciertos casos existen paradojas. Sin embargo, prevalece la generación y necesidad de seguir ampliando las herramientas, procesos y elementos que constituyen un respectivo saber en cualquier área del mundo de las ciencias. La llegada del internet (red de redes) simbolizó un despliegue transcendental por intentar superar las barreras de un tipo de información mal denominada "selectiva" o cerrada, lo que permitió una especie de liberalización y/o divulgación del conocimiento en red.

En efecto, la tecnología en el campo de la informática ha pasado a ser un espacio de suma importancia al interior de la ciudadanía. La promoción de ampliar los canales comunicativos y su relación con la cultura de lo público ha pasado a ser un punto esencial en los debates modernos sobre la sociedad civil y la democracia en sus respectivas dimensiones. 
La política, la economía y la cultura como facultades humanas también han sufrido modificaciones a lo largo de la historia de la humanidad. A través del desarrollo local, económico, político y social en lo individual/colectivo se ha generado un "progreso" de la capacidad de comunicación e interacción entre los sujetos, llegando al punto de procesar y distribuir de forma lineal o no-lineal algún tipo de conocimiento conformen a las necesidades de la población. De esta manera, las Tecnologías de la Información y las Comunicaciones TIC, están en todos los ámbitos y esferas de la vida del ser humano, en lo social, lo político, lo económico, lo educativo y lo cultural entre otros; es decir que no existe ningún campo del saber donde las TIC no constituyan una forma de interacción virtual de gran importancia. Aunque en la actualidad se ha procurado avanzar en la difusión y el acceso al internet, la aplicación de herramientas propias de las TIC en los diferentes entornos sociales, todavía se logran apreciar pocas estrategias adecuadas por parte de las instituciones oficiales, privadas, centros e institutos educativos encargados de la aplicación de las tecnologías de la información a los espacios correspondientes de cualquier tipo de población (infantil, juvenil, adulta y tercera edad).

La emergencia de las tecnologías de la información y las comunicaciones en el presente siglo, según los investigadores Eduardo Sandoval y Laura Mota se constituye en un proceso de larga duración debido al crecimiento estratégicos y desarrollo estructural por parte del Estado frente a la sociedad civil sin dejar a un lado la lógica mercantilista producto de los monopolios del poder político orientados a la enajenación de los medios de comunicación en los distintos países de Latinoamérica, tal como sucede en México y Colombia a través de la transnacionalización de las telecomunicaciones al servicio de las empresas multinacionales.

El amplio debate de incorporar y/o reconocer una política de Estado sobre las TIC representa un campo de oportunidad que puede articularse con el desarrollo estructural de un país, así pues, la necesidad de establecer los derechos y servicios necesarios y acordes a las necesidades de la sociedad civil se identifica como un reto por parte de las autoridades competentes en los distintos países con el fin de impulsar programas, proyectos y actividades orientadas al uso de la TIC para la formación ético- política de la ciudadanía. 
Para Castells (2001) uno de los factores, que ha influido en que esta revolución tecnológica se desarrollara vertiginosamente, ha sido el comportamiento de los innovadores que la crearon, ya que sus mentes creadoras, a decir motivadas por la pasión y la codicia, se han preocupado constantemente de estar alertas a las señales y necesidades crecientes del mercado de usuarios, buscando nuevos nichos de necesidades que satisfacer con nuevos productos y procesos.

Para entender dicho proceso, el paradigma tecnológico propuesto por este autor, considera los siguientes aspectos:

1. La información es su propia materia prima, son tecnologías diseñadas para actuar sobre la propia información.

2. La información ha pasado a constituir parte integral de la actividad humana, por esto se destaca la capacidad de penetración de los efectos de las nuevas tecnologías.

3. Todo el sistema se basa en la lógica de la interconexión, estableciéndose una nueva topología: la red.

4. Los procesos, las organizaciones y las instituciones se adaptan a la flexibilidad de las TIC, ya que se exponen a frecuentes reordenamientos de sus componentes.

5. Existe una convergencia creciente de tecnologías específicas en un sistema altamente integrado (Castells, 2010).

De esta forma, la finalidad del presente artículo consiste en exponer los elementos teóricoanalíticos que constituyen la importancia de las TIC como herramientas para superar por medio de la educación para lo público algunos fenómenos del conflicto armado. Buscando así, mencionar la conexión entre las TIC con la construcción de paz territorial teniendo en cuenta el contexto de un postacuerdo y/o postconflicto ${ }^{1}$.

\footnotetext{
${ }^{1}$ Es importante señalar que no haremos discusión sobre el uso de la categoría postacuerdo o postconflicto dado que sería ampliar otra ventana de discusión, lo que nos interesa es situar a los/as lectores para que identifiquen que la investigación está inmersa en un escenario de conflictividad como lo constituye la amplia historia de la violencia en Colombia.
} 


\section{Desarrollo}

Hoy, el adecuado dominio en el manejo de la información, es clave en todos los sectores de la producción. Al respecto Velásquez (2006) sostiene que la sociedad actual, conocida como la sociedad del conocimiento, se caracteriza por la gran disponibilidad y fluidez de la información, a la cual deberían tener acceso todos los seres humanos que la requieran o necesiten; la calidad de la misma será juzgada a criterio de quienes la usen y de sus capacidades y conocimientos para discernir que es bueno y que no, qué información es relevante y cuál no.

El internet ha tomado fuerza en diversos contextos de la sociedad en donde se ha llegado al punto de reconocer como las actividades cotidianas han sido permeadas por la influencia del mundo de la red, esta situación podría reconocerse como un estado de naturalización del internet, aunque se logre apreciar altos niveles de desigualdad en términos de acceso y servicio que ofrece las telecomunicaciones en el país.

Así pues, la larga historia de la violencia de naturaleza armada en Colombia, resultó ser un gran vacío de poder político por parte de las élites en su afán de imponer un orden al servicio de los intereses del capital. El uso de la violencia, la acumulación de tierras y la necesidad de configurar un imaginario de antagonismo entre dos sectores tradicionales (liberales y conservadores) en los años 50 fue la etapa en que se cimentaron las bases para un conflicto de larga duración con gran fuerza en las regiones más apartadas del territorio.

Tal como lo señala Zuleta, E y Valencia, A (1991) y Sandoval (2003) el fenómeno de la violencia responde a la construcción de un imaginario basado en la negación del otro, y la necesidad de desconocer la "otredad" como un referente de diálogo en sí mismo, dicha situación se ha visto como un punto característico de la generación de racismo, sexismo, discriminación y xenofobia locales, regionales y nacionales dentro del territorio colombiano llegando al punto de simbolizar una disputa por el poder político sobre los territorios.

El panorama de corrupción, clientelismo y acumulación de poder de la mano de la intensificación de la carrera armada por parte del gobierno y la organización al margen de la ley se ha convertido en un punto fundamental que complejiza el conflicto armado del país, dicha situación se constituye como un factor determinante por analizar la pugna entre la 
lógica estatal y la acción de los grupos armados ilegales.

Al mismo tiempo, la amplia desigualdad social, los bajos niveles de educación crítica y la poca voluntad política de generar procesos desde adentro han conllevado a los grupos oprimidos y/o subalternos en una posición de exclusión de amplia magnitud, una serie de contextos de violencias sistemáticas sumado la débil presencia de la institucionalidad en los problemas sociales, políticos, económicos y culturales en las zonas mal - llamadas periféricas.

La compleja realidad de las naciones de Nuestra América no es ajena a la violencia, por el contrario, se subsumen entre las contradicciones del poder, el capital y la política proveniente de las élites criollas y la imposición de un capitalismo tardío que ha constituido múltiples conflictos en todos los grupos sociales, en relación a esto los avances de la ciencia, la técnica y la tecnología también contribuyen a la generación de "otras" perspectiva sobre la violencia, el conflicto, la política y los intereses generalizados de diferente partes sobre las consecuencias del conflicto armado (Sandoval, 2012) y (Capera; Ñañez, 2017).

Por eso, en la era industrial la maquinaria y la mano de obra calificada, unidos al aspecto administrativo y conocimiento en la información de los mercados constituyeron un aspecto clave en los modos de producción, estos elementos posicionaron el área de servicios como una parte fundamental para las empresas en el juego de la oferta y la demanda. Otras de las características de la era industrial es que el hombre tiene acceso a los bienes producidos por otros; por su parte en la sociedad post - industrial se tiene acceso a servicios generados entre diferentes actores (agua, luz, teléfono, etc.), mientras que en la sociedad de la información se tiene acceso a la información generada por otros (bases de datos, bibliotecas, redes, etc.).

Las Tecnologías de la Información y las Comunicaciones, conocidas comúnmente como TIC, es una sigla que se utiliza para hacer referencia a una gama amplia de servicios, aplicaciones y tecnologías que utilizan diversos tipos de equipos (hardware), y que a menudo se transmiten a través de un entramado de redes de comunicaciones (software). Las TIC son utilizadas como instrumentos que ayudan en la obtención de información y que una vez se haya utilizado esta información para construir nuevos conocimientos, se pueden generar innovaciones. 
En este sentido, el Internet para Castells (2002) representa un tejido de las vidas de los seres humanos dado que posibilita la comunicación por medio del ambiente virtual, a esto la interacción se transforma constantemente llegando al punto de formar redes societales, es decir, mecanismos de amplia construcción, divulgación e información de algún tipo de conocimiento. La razón del Internet está vinculada al espectro de aceptación y recepción del mensaje que se pretende dar a conocer / difundir.

La razón de ser de la tecnología debe ir más allá de una visión simple, consiste en reconocer su capacidad de cohesión, organización y difusión del saber, lo que significa un medio para generar interés entre cualquier grupo de la sociedad civil. Por ello, la apuesta de fortalecer las TIC simboliza todo un reto por intentar superar las formas tradicionales de comunicación y dar paso a prácticas tradicionales de interacción y relaciones comunicativas entre los sujetos.

Por su parte, el gobierno colombiano al conocer los avances dinámicos de la era de la comunicación y la sociedad en red, se propuso ante las organizaciones internacionales y la Unesco incentivar una política pública sobre las TIC y su aporte al desarrollo del país, apareciendo así elementos como:

1) El acceso e infraestructura de la información y telecomunicación al nivel nacional, la cual tenga un bajo costo en materia del uso de las TIC. 2) las campañas de educación y sensibilización de las TIC en los procesos educativos, políticos y culturales en distintos espacios sociales. 3) El uso privado por parte de las empresas para impulsar la competitividad entre el sector público y privado. 4) La generación de apoyos en el contenido y servicios en línea. 5) La construcción de una cultura virtual del respeto, la transparencia e idoneidad sobre los asuntos del gobierno y la sociedad civil (Balen, 2016).

Frente a esta postura institucional se logra evidenciar que en la realidad han sido muy escasos los avances sobre estrategias idóneas que permitan la generación de una cultura del conocimiento a través de las TIC; esta ha sido visto como un instrumento de doble rasero dado el enfoque que utiliza los actores al interior del gobierno con el fin de establecer un tipo de información y a quien se encuentra dirigida. 


\section{Paz, educación y TIC}

La sociedad de la información se puede representar como un modelo conceptual, que se representa tres ejes: uno horizontal, uno vertical y uno diagonal; en el superior, se encuentra la infraestructura, servicios genéricos y habilidades y destrezas; en el vertical, sectores de la sociedad afectados por las TIC (gobierno, comercio, salud y educación) y diagonal (regulación y legislación, financiamiento e incentivos).

Por ende, el uso de las TIC con fines educativos de manera que garanticen su apropiación y uso correcto y al mismo tiempo propicien la inclusión social, implica tener en cuenta elementos como:

a) Organización de infraestructuras para el óptimo uso de estas tecnologías.

b) Dotación de equipos y actualización permanente de los mismos.

c) Formación de talento humano y actualización constante del mismo.

d) Voluntad política (Velásquez, 2006).

A menudo se toman decisiones respecto al uso de las TIC, más relacionados con aspectos económicos, de moda, o incluso por conveniencia personal, administrativa, más que por las potencialidades que estas ofrecen y en alguno casos se desconoce el potencial que significa una sociedad informada de manera crítica y autocrítica, es decir, la existencia de un vacío de profesionalización, educación y formación en el uso y desuso de las TIC se encuentra presente en las dinámicas de la sociedad colombiana en medio de un proceso que busca superar las secuelas de un conflicto armado de más de medio siglo.

De acuerdo con la Unesco y el Ministerio de las Tecnologías de la Información y las Comunicaciones de Colombia, la necesidad de superar la brecha tecnológica y los bajos niveles de educación basada en las TIC, pasó a ser un talón de Aquiles debido a los requerimientos que le exigieron las organizaciones internacionales, el FMI, BM y ONU al gobierno nacional construir programas acordes a las necesidades del mercado corporativo con el fin de incentivar el uso de la producción y las herramientas de las tecnologías.

Este panorama refleja la necesidad de integrar de forma adecuada las TIC a los contextos cotidianos de la sociedad colombiana, para así lograr un aprendizaje significativo y una forma 
efectiva de aprender aprendiendo por medio de los programas y las aplicaciones entre el gobierno (línea) y las prácticas de los grupos sociales con el fin de buscar superar desde adentro y por medio de la comunicación virtual algunos aspectos producto de la violencia en Colombia (Cárdenas; Tovar, 2011).

Es importante proponer modelos alternativos de educación con las TIC enfocadas a localizar las necesidades/demandas que poseen los grupos sociales del país, se constituyen como un instrumento de larga duración que va en consonancia a reorganizar las formas de comunicación entre el ciudadano y las instituciones, por ello, los medios de comunicación reflejan un campo de oportunidad de formación para lo público.

El asunto radica en proponer prácticas pedagógicas desde una concepción crítica, lo que implica apostarle a repensar modelos de enseñanza desde los distintos niveles educativos (básica, media, técnica, tecnológica y superior), para así lograr construir imaginarios en común que pueda ser rutas de interacción y cohesión de proyectos territoriales, tal como se puede concebir con la configuración de las herramientas tecnológicas en función de los intereses/necesidades del ciudadano en su respectivo contexto sociocultural.

Tal como lo argumentan Sandoval, R y Alonso, J (2015), al posicionar un pensamiento crítico desde el sujeto, es decir, establecer un reconocimiento de la diferencia subjetiva y la praxis individual o colectiva sobre el territorio, aquí la emergencia de asumir una postura reflexiva - transformativa responde a una necesidad de hacer del pensamiento una forma de práctica en sí misma.

Por ende, el papel del ciudadano en las TIC consiste en un ethos que posibilite la construcción de un pensamiento crítico desde abajo, para así lograr apropiarse de dichas tecnologías como herramientas alternativas no mercantilizadas y funcionales a los intereses sistémicos, tiene que ver con la importancia de desconfigurar los esquemas planteados a partir de una visión lineal/vertical del Estado hacia la sociedad civil.

Parte de dicho paradigma responde a lo que Sandoval (2009) ha desarrollado como una mirada alternativa por desprenderse de los modelos sistémicos y descontextualizados de las TIC sobre la sociedad civil, y dar un giro alternativo que establezca una mirada horizontal en donde los programas, proyectos, cursos y diplomados sean congruentes con una pedagogía de paz a través de actos y escenarios simples como son por ejemplo las redes sociales, buscando incentivar estas prácticas pedagógicas en los contextos institucionales y no- 
institucionales para construir un ambiente de acercamiento con los principios, propuestas, componentes y fines que constituyen el postconflicto desde las regiones y los territorios más vulnerables/desconectados del país.

La lógica de impulsar un escenario de formación crítica tiene que ver con una pedagogía en el mismo sentido, lo que representa un marco de oportunidad por concebir un sujeto con capacidad de apropiarse de forma reflexiva y propositiva sobre la política, la economía, lo social y la cultural que son elementos que influyen en la construcción de la paz en medio de un ambiente de postconflicto, a su vez, la iniciativa de concebir la educación como un campo de oportunidad para superar el rezago informativo crítico y subsanar los vacíos de la nube de desinformación creada/promovida por los medios sistémicos al servicio de los grupos hegemónicos que tienen una práctica discursiva en contra del proceso de paz en sus distintas acepciones sobre las regiones.

\section{Algunas perspectivas de uso de las TIC en el posconflicto}

El uso y apropiación de las TIC como herramienta de alfabetización científica y tecnológica requiere de voluntad política del gobierno nacional y del papel protagónico de la sociedad. En este orden de ideas la universidad colombiana debe convertirse en un facilitador de los procesos de cambio e inclusión que demandará el posconflicto. En tal sentido vale la pena considerar los siguientes aspectos de suma importancia consiste en aprender a: comprender, hacer, ser y convivir. A continuación, se plantean los principales aspectos a considerar para propiciar el uso de las TIC como mecanismo de inclusión para el posconflicto en la República de Colombia (Velásquez, 2006).

Entre las principales perspectivas de las TIC como herramienta de inclusión social para posconflicto se resaltan:

a) Crear comunidades de aprendizaje

b) Generar propuestas orientadas a humanizar la educación.

c) Propiciar la organización de redes de aprendizaje

d) Contribuir al fortalecimiento de plataformas virtuales de aprendizaje

e) Conformar un banco de buenas prácticas productivas que consoliden la formación de competencias laborales en las regiones. 
f) Propiciar el diseño y desarrollo de programas para emisoras comunitarias, orientados al fortalecimiento de valores, la convivencia pacífica, el respeto por la diferencia; de la mano con el gobierno, tales como narraciones sobre conciliación pacífica de conflictos.

Esta serie de propuestas se articulan con la concepción de una ciudadanía crítica, autocrítica y reflexiva, tal como lo considera Márquez Fernández (2013), Freire (2014) y Capera, y Ñañez, (2017), la apuesta de pensar desde abajo la democracia significa un giro radical sobre las formas tradicionales de concebir el poder, la política, la cultura y la economía; resulta ser un paso en donde la ciudadanía y lo público se encuentran en constante interacción en todo los campos educativos, formal, informal, familiar entre otros. Esta situación puede ser asimilada como una manera de generar enclaves democráticos subalternos por medio del uso significativo de las TIC en los contextos locales, regionales y nacionales.

Con respecto a esta lógica, las TIC en el posconflicto se convierte en un escenario de vital importancia para agitar y demostrar que los elementos tradicionales de comunicación se encuentran en crisis, y que por ende la sociedad del conocimiento virtual no debe ser homogenizada a un tipo de sociedad del consumo y/o mercantilización, sino que representa un campo de oportunidad por problematizar, educar, sensibilizar y reflexionar sobre los impactos de cualquier naturaleza que ha dejado un conflicto armado de larga duración sobre los grupos más excluidos de la sociedad colombiana.

Alguno de los principales aspectos a considerar acerca del papel de las TIC en el posconflicto, consiste en:

\section{Aspectos administrativos}

a) Conformación de un grupo interdisciplinar de expertos en uso y apropiación de las TIC

b) Censo virtual, para identificar actores y necesidades.

c) Análisis de perfiles de los actores beneficiados (edad, nivel de escolaridad, intereses formativos, etc.).

d) Bancos de empleos específicos para los actores. 
e) Organización de redes de emisoras comunitarias, que contribuyan a socializar los logros, propuestas y vinculación de actores en diversas actividades(Velásquez, 2006) y (Cárdenas; Tovar, 2011).

\section{Aspecto formativo.}

Desarrollo de plataformas específicas para la formación en competencias laborales, mediante estrategias: E-learning, M- learning, B-learning, Creación de centros de innovación en las regiones para diversos actores y sectores, adquisición y uso de bases de datos para diversos actores y sectores, desarrollo de OVA y aplicaciones para diversos actores y sectores.

\section{Aspecto social.}

a) Creación de círculos de experiencias para solución de conflictos.

b) Visualización de la verdad a través de medios masivos de divulgación como las redes sociales,

c) creación de objetos virtuales de aprendizaje y socialización orientada a resolver problemas regionales.

4. Aspecto laboral para los actores.

a) Organización de microempresas orientadas al desarrollo de competencias laborales y oficios que las TIC generan como instalación de equipos mantenimiento, vigilancia de infraestructura en regiones de presencia de los participantes en el conflicto.

b) Aprovechamiento de experiencias productivas de los actores que beneficien a sectores vulnerables.

En el plano técnico las TIC podría enfocarse en el escenario del posconflicto por medio del uso consensuado de las herramientas de educación virtual y formación ciudadana en lo público, a su vez, la posibilidad de organizar procesos socio-políticos diseñados en temas económicos y culturales enfocados a grupos sociales (indígenas, negritudes, campesinos, mujeres, estudiantes entre otros). Una forma de reconocer como la educación se constituye en un campo de superación de las prácticas de violencia, homogenización y linealidad por 
re-producir formas y estrategias tradicional del conocimiento, ya con la llegada de las TIC se requiere una modernización estructural que sean conducente con las necesidades de las sociedades en sus respectivo tipo de naturaleza.

En esto tiempos donde se ha podido reconocer un acercamiento institucional entre la guerrilla de las Farc $^{2}$ - ep con el gobierno nacional, las TIC pasan asumir un papel transcendental en su tarea de informar, educar y difundir un tipo de conocimiento sobre un tema en particular como resulta ser los acuerdos de paz negociados en la Habana Cuba, esta situación ha vivido una serie de especulaciones, distracciones y noticias erradas por parte de distintos grupos contrarios a la posición gubernamental de empezar algún tipo de comunicación con esta guerrilla debido a lo que significa en el imaginario de los colombianos.

Tal como se reconoce el advenimiento de las Tecnologías de la Información y la Comunicación (TIC), se han reevaluado como un campo para superar las fronteras físicas y dar paso al mundo del acceso al internet, la comunicación virtual y la interacción online, ha conllevado a la invención de múltiples contextos en donde la conectividad paso a ser un tema indispensable en el desarrollo de una nación.

Por su parte, la sociedad de la información es la muestra del mundo modernizado y reconfigurado por el uso extensivo del Internet y las aplicaciones en la red, esta se constituye como un simbolismo cultural y una forma dinámica de ir más allá de las estructuras y organizaciones tradicionales en nuestra época (Sandoval; Mota, 2007). La necesidad de utilizar las TIC para superar la marginalidad educativa, social, cultural y política es un reto que requiere de elementos colectivos y cooperativos por parte de distintos actores de la sociedad, así los establecimientos educativos son espacios idóneos para incentivar el uso adecuado, racional, emocional y sentípensante de las TIC para ir superando desde la divulgación de un tipo de información transparente los elementos centrales que constituyen los acuerdos de paz y la oportunidad de reflexionar desde el postconflicto (Velásquez, 2006).

La propuesta desarrollada por Velásquez (2011) en su libro sobre la didáctica de las ciencias, la educación científica y el papel de las TIC en la educación superior, nos muestra

\footnotetext{
${ }^{2}$ Fuerzas Armadas Revolucionarias de Colombia se constituyó en el grupo guerrillero más longevo de Latinoamérica.
} 
herramientas necesarias en el tipo de formación que se viene realizando en contextos locales, y a su vez nos resalta porque es necesaria invertir, impulsar y fortalecer las TIC como herramientas de enseñanza en una sociedad globalizada, a este análisis se puede sumar el contexto temporal y espacial que vive la sociedad colombiana dentro de un conflicto diseminado y complejizado en las territorios.

Por eso, la construcción de paz territorial y su relación con las TIC nos permite pensar en una sociedad democratiza y con criterios autonómicos que le facilite la conciencia crítica de la ciudadanía, al momento de legitimar o desconocer un aspecto de interés público. Dicha situación también representa un campo de oportunidad por incluir políticas y programas educativos pero en función de los grupos excluidos para así fortalecer el componente de la enseñanza virtual como un aspecto de las democracias subalternas (Sandoval, 2012) y (Márquez, 2013).

Las nuevas condiciones de trabajo y la dinamización del capital en materia de productividad, ha gestado todo un desarrollo de las tecnologías como una forma de impulsar la lógica de modernización y expansión de las formas productivas del Estado, dicho panorama se ha convertido en un elemento propicio para constituir interacciones sociales, políticas, económicas y culturales sobre la población de un país. El panorama colombiano que se ha caracterizado por un conflicto estructural y sinérgico de violencias que ha repercutido en los sectores más vulnerables de la nación. A esta lógica, se le suma la aplicación de un modelo político - económico basado en la negación del "otro" y la preferencia a la mercantilización del saber en consonancia a los intereses privados del capital (Capera; Nañez, 2017).

Por ende, aludimos en reconocer que la "brecha digital" puede ser un discurso utilizado por ambas partes orientado a fortalecer los vacíos de una educación de la información masiva o la aplicabilidad de un tipo de conocimiento restringido y manipulado por los actores estatales, así pues pensar en que aspectos aporte las TIC a la construcción de paz territorial en Colombia, significa reconocer cambios de las formas de hacer política y concebir las relaciones sociales entre los distintos sectores del país, es la muestra por considerar importante la TIC como instrumentos para impulsar la ciencia, la técnica, la cultural, la participación política y los imaginarios sobre una democracia más informativa y autocrítica de sí mismo. 
El panorama social y económico de la nación nos exige reconocer la heterogeneidad estructural que viven los sectores sociales en sus múltiples realidades. Así los procesos de apertura de un conocimiento más amplio y un saber más democratizado se convierten en un reto al interior del uso masivo y transparente de las TIC para la educación ciudadana. Tal como lo señala Velásquez (2006) y Balen (2016) cuando parten de asumir que el escenario complejo del postconflicto nos exige soluciones y/o alternativas complejas a esta misma situación, por ello, las TIC no pueden ser vistas como una política o un espacio de tercera categoría sino como una oportunidad para ir abonando elementos para la reparación, la rearmonización, la resistencia y las verdades por medio de las condiciones que ofrecen las tecnologías en las actuales sociedades (Sandoval; Mota, 2007).

Teniendo en cuenta la necesidad de ir superando el conflicto armado en sus impactos negativos sobre los grupos sociales, las TIC pasan hacer un mecanismo de interacción amplia dado que permiten una mayor conexión y sociabilidad de las propuestas en común para canalizar fuerzas entre los actores, siendo así, que para usarla y poner en función las TIC se requiere condiciones en el desarrollo de las personas y el nivel de educación y formación con el fin de lograr un proceso exigente o adecuado a su condición real.

El proceso del Internet en estos tiempos parece ser un elemento irreversible dado que la necesidad de comunicarse y estar en relación con los avances, acontecimientos y novedades del último instante son cada vez necesario, a esto se suma el boom de las redes sociales y la constante difusión de noticias en los medios alternativos permiten contextualizar el porqué de las TIC en un tipo de sociedad moderna. En el caso colombiano se puede reconocer la necesidad de informar y garantizar la no -manipulación del tipo de contenido en particular aquellos relacionados con el postconflicto.

Asimismo, una manera de poder hacer peso al centralismo y la concentración del poder “informático" según Castells (2002) tiene que ver con la superación de los monopolios informáticos y la subordinación por parte de los gobiernos frente a los sectores dueños del gran capital. Esta situación ha permitido ampliar la brecha de exclusión y los intereses político- mercantiles de enajenar las TIC como instrumentos al servicio de los intereses de ciertos sectores público y privados. Una situación que debería verse de otra forma dado que la información por naturaleza y principio se caracteriza por un diseño de redes pluralistas, 
abiertas y libres en donde se pueda ejercer proyectos adecuados a las condiciones estructurales del grupo social del país (Velásquez, 2011 ).

Los medios de comunicación formales y alternativos juegan un papel central en su desafío de ir construyendo otra realidad donde la información logre ser más parcial y objetiva en un sentido crítico, aquí las TIC pasan a ser un factor determinante en la sociedad red dado que le facilitan hacer común temas que se veían tan complejos para cualquier ciudadano con un nivel particular de formación sin dejar a un lado la capacidad de llegar y ser reproducida la intercomunicación dentro del internet y el uso de las redes sociales como medios para difundir un tipo de noticia.

Siendo importante reconocer la poca experiencia del gobierno y su falta de voluntad política por impulsar y fortalecer las TIC como un mecanismos de divulgación sobre los intereses reales que conforman los acuerdos de paz y la importancia de transitar por un postconflicto, sin olvidar la iniciativa de formar redes, equipos y campañas de capacitación del internet y sus herramientas con el fin de establecer rutas coherentes con los sectores vulnerables y víctimas del conflicto armado (Capera; Nañez, 2017).

La falta de establecer políticas públicas reales y coherentes con los territorios, es una déficits del gobierno nacional en su responsabilidad pública dado que no se cuenta con los suficientes elementos materiales e inmateriales para llevar a cabo procesos de corta y larga duración en el campo de la formación para lo público y ciudadana desde las TIC, esta situación ha despertado una serie de divergencias con actores oficiales y privados que reconocen que parte de la apuesta a la paz requiere de una investigación en ciencia, técnica y tecnología en diferente ámbitos de la vida socio-política.

Así pues, logramos reconocer que la construcción de paz territorial es un enfoque alternativo y de naturaleza popular, puesto que considera importante ver la problemática desde abajo y de forma horizontal, es decir establecer un giro sobre la visión normativa, institucional y lineal de concebir la paz como un modelo ejercido por la institucionalidad, en este caso se da preferencia a lo generado por los sectores populares, subalternos y oprimidos que por medio de su praxis realizan acciones que son viables para asumir las TIC como un espacio de oportunidad e interacción más informado, viable y parcializado para así demostrar que la formación y el conocimiento verídico es parte de un derecho fundamental que debe ser contemplado en el acuerdo de paz y la democracia en el postconflicto. 
La pertinencia de ir contracorriente es la respuesta de establecer "otras" lógicas de sociabilidad del conocimiento y la era de la información, tiene como principio establecer las mínimas formas de interacción y legitimidad que debe existir en la democracia, las formas de construir paz desde abajo y la acción de diferentes sectores desde las regiones, aquí las TIC pasan a ser un elementos decisorio y fundamental en el reconocimiento de que tipo de información, programa y educación virtual o informática se desea establecer en un momento tan emblemático como es el postconflicto.

Una manera de problematizar el debate y ponerlo a la luz pública como un tema de interés general, de naturaleza pública y de gran pertinencia en el ámbito local, nacional e internacional que ponen al Estado Colombiano en su diatriba de generar las mínimas condiciones de uso y estrategias de las TIC como instrumentos idóneos en la superación del conflicto y la necesidad de una paz estable, duradera y desde abajo que no sea ajena a los intereses reales de los territorios que pretende dar un giro a las historia de violencias, conflictos y luchas por el control sobre las regiones por parte de la guerrilla frente a la militarización del Estado en los distintos departamento del país.

\section{Metodología}

La metodología utilizada en el presente artículo de investigación consistió en un análisis crítico sobre las siguientes categorías: TIC, posconflicto, violencia, construcción de paz y territorialidad. La pretensión fundamental consistió en señalar como el actual panorama de diálogos de la Habana para el año 2015 pasó a politizar la sociedad colombiana, llegando al punto de crearse imaginarios, nociones e ideas ajenas a la naturaleza propia de las negociaciones.

Dicha situación, implicó conocer que concepción tiene el gobierno nacional sobre las TIC y si existe una postura propia sobre el uso de estas en el postconflicto, encontrando que la planificación estructural no contempla un espacio adecuado sobre la importancia de las tecnologías de la información como una pieza fundamental en su labor de divulgar, informar y capacitar a la ciudadanía en un tema tan estructural como significa la paz desde los territorios. 
En segunda medida, se recurrió a rastrear los documentos institucionales (oficiales) nacionales e internacionales que vinculen propiamente al Estado colombiano por medio de sus instituciones a garantizar las mínimas condiciones del uso de este tipo de tecnologías de información. Aunque los archivos recopilados se logra reconocer un interés bajo sobre este campo y su afinidad de construir la paz en un momento de postconflicto, es el reflejo del poco interés e inversión en fortalecer este campo de interés público.

En tercera medida, se procedió a la construcción de herramientas analíticas que lograran reconocer que tipo de programas, proyectos o planes existen sobre las TIC y como ha sido el enfoque utilizado y sobre qué tipo de población, aquí se logró encontrar dos programas esenciales Computadores para Educar y Gobierno en Línea, el primero orientado a la población infantil y juvenil de las distintas instituciones educativas y el segundo respondía a un interés por buscar agilizar los procesos político - administrativos por medio de la web. En este sentido, se carece de un programa coherente y congruente con lo que significa el postconflicto teniendo en cuenta la naturaleza real de los acuerdos entre la guerrilla y el gobierno nacional, y la oportunidad que tiene la sociedad en general de empoderarse y generar formas alternas de construcción de paz desde abajo.

Un aspecto del proceso de investigación consistió en la poca información vinculada a este tema y desde una perspectiva crítica latinoamericana, es decir, falta herramientas que problematicen este tema tan reciente y pongan en el escenario académico y social las oportunidades que se generan sobre la aplicación de programas y/o proyectos acordes a los intereses reales del acuerdo de paz para lograr transitar a un escenario de postconflicto en el país. En este sentido, la importancia de seguir investigando esta situación adquiere relevancia dado los últimos avances en materia de sociedades en movimiento y la cultural de la red que interconecta distintos actores sociales y grupos de la nación.

Por último, en medio de la sistematización de la información se pudo hallar la necesidad de seguir profundizando en la temática de las TIC como instrumento de cohesión social en el escenario del postconflicto, a su vez, la importancia de fortalecer espacios locales, regionales y nacionales en donde se logre construir un ambiente de crítica, autocrítica, 
reflexión y propuestas desde adentro para mostrar otra forma de construcción de paz desde abajo y al servicio de las necesidades de los grupos oprimidos.

\section{A modo de conclusión}

Las TIC simbolizan un espacio de integración, educación y formación para lo público, tiene una carga valorativa en función de formar la ciudadanía en un sentido amplio con elementos para que se logren integrar con la sociedad en red/movimiento. A su vez, refleja todo un corpus de oportunidad de gran importancia al tener acercamiento por medio de la red a distintos sectores de la sociedad.

El postconflicto representa un escenario de construcción colectiva y desde debajo de procesos de liberación, se podría pensar en la posibilidad de establecer elementos para difundir y educar virtualmente sobre las necesidades reales que requiere la sociedad teniendo en cuenta la oportunidades y contradicciones de la paz en Colombia. Igualmente, se puede reconocer la posibilidad de profundizar en el tema más allá de la visión institucional para dar paso a las prácticas colectivas de conectividad al servicio de los intereses de los grupos subalternos.

El componente de la educación es de vital importancia si se asume un proyecto de indagación, divulgación y formación sobre el postconflicto, ya que la construcción de la paz territorial está vinculada a la necesidad de subsanar las necesidades/demandas de las comunidades a partir de sus propias experiencias, es decir, que la relevancia de conocer su contexto sirve como instrumento para implementar de forma horizontal los programas, proyectos, cursos y medios a través de las TIC para que sean congruentes con sus especificidades en el territorio.

La lógica de reconocer un sujeto crítico, autocrítico y reflexivo está asociada a la apuesta por apropiarse de la paz como un derecho y un deber público, aquí la superación de las ideologías, los personalismos y la fragmentación social sobre el tema, se asume como un reto por construir rutas pedagógicas que generen integración, unidad y dialogicidad entre distintos actores, pero enfocados a construir la paz desde el pluriverso de realidades en sus contextos territoriales. 
Al mismo tiempo, es importante resaltar aspectos como: A) se requiere decisión política para que las TIC jueguen un papel protagónico para los diferentes actores del postconflicto particularmente para la educación, principalmente en lo relacionado a inversión económica para sectores vulnerables en la lógica de democratizar la información virtual. B) una nueva generación de líderes que comprenda el papel de las TIC como herramienta de inclusión para los diferentes actores del postconflicto. C) las universidades deben ser actores clave que contribuyan a facilitar el uso y apropiación de las TIC en la solución de diferentes problemas regionales. D) la importancia de ampliar la inversión para adquisición de bases de datos, dispositivos móviles, masificación del internet de banda ancha. E) la inversión en mejores vías para que los atores puedan comerciar sus productos obtenidos en los cultivos con la apropiación y uso de las TIC.

La configuración de líneas educativas que pongan en interlocución la paz, la educación y las TIC tiene que ver con la edificación de un diseño subalterno que ponga al sujeto y la comunidad como actores fundamentales, los cuales entran en diálogo con las instituciones, pero creando diferencias en medio del proceso de interacción con las tecnologías y en particular sobre la construcción de paz territorial para asumir una postura sobre el contexto sociocultural de la ciudadanía para lo público.

Por último, esta serie de propuestas son un principio para dejar abierto el debate sobre complejizar las TIC, y pasar de un plano lineal y positivista a una visión más desde la interacción socio-política o una lógica crítica en donde se hagan propuestas alternativas de comunicación que superen los intereses del capital privado, asimismo las formas tradicionales de dominación ejercidas por los sectores transnacionales que en la actualidad son los dueños de los medios informáticos y el uso libre, sin discriminación y transparente del internet en la sociedades en movimiento.

La sociedad colombiana en el postconflicto requiere mayor acción dialógica, interacción comunicativa y democratización de las TIC para así permitir la generación de un tipo de conocimiento amplio, y que logre llegar a los distintos sectores sociales del país. Al mismo tiempo, representa un reto para articular la cultura de paz desde un enfoque intercultural, es decir la construcción de paz desde abajo y en consonancia a los intereses de 


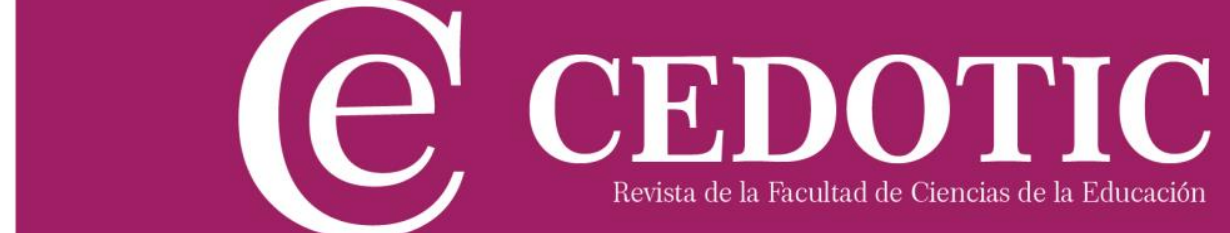

los grupos subalternos, lo que permitirá la apropiación de espacios virtuales que fortalezcan la formación de lo público y la construcción de un tipo de ciudadanía crítica, autocrítica y reflexiva de los fenómenos socio-políticos de la época. 


\section{Bibliografía}

Balen, C. (2016). Las tecnologías de la información y las comunicaciones en el postconflicto colombiano. Revista de Ingeniería, no $44,40-45$.

Capera, J \&Ñañez, J. (2017). Una perspectiva crítica sobre la violencia en Colombia en épocas del postconflicto. Utopía y Praxis Latinoamericana, 78(22), 153-162.

Cárdenas, N \& Tovar-Gálvez, J. (2011). Computadores y red en Colombia: posibilidad de interacción globalizadora en instituciones educativas públicas y desarrollo regional. Pixel-Bit. Revista de Medios y Educación (38), 177-186.

Castells, M. (2002). The Internet galaxy: Reflections on the Internet, business, and society. United Kingdom: Oxford University Press on Demand.

Castells, M. (2010). The Information Age: Economy, Society and Culture. John Wiley \& Sons Ltd: Wiley - Blackwell.

Freire, P. (2014). Pedagogy of hope: Reliving pedagogy of the oppressed. London: Bloomsbury Publishing.

Márquez Fernández, Á. (2013). Crisis ideológica de la democracia liberal: representación social y legitimidad política. Encuentros, 11(2), 5, 67-76.

Sandoval, E \& Mota, L. (2007). Indígenas y democracia en las tecnologías de información y comunicación (TIC). Presentado en VI Jornadas latinoamericanas de estudios sociales de la ciencia y la tecnología, 1-27.

Sandoval, E. (2003). Violencia y paz imposible para los indígenas en Colombia. En E. \&. Sandoval, América Latina: conflicto, violencia y paz en el siglo XXI (págs. 106-125). México: Insumisos Latinoamericanos/Libros en Red.

Sandoval, E. (2009). Ciudadanía universal, derechos políticos y paz en la migración. En V. Martínez Guzmán, \& E. A. Sandoval Forero, Migraciones, conflicto y cultura de paz (págs. 19-45). México: Bonobos Editores. 


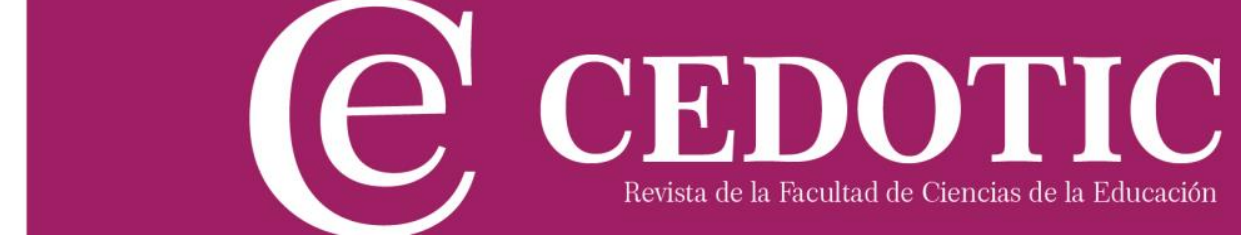

Sandoval, E. (2012). Estudios para la paz, la interculturalidad y la democracia. Ra Ximhai, 8(2), 17-37.

Sandoval, R \& Alonso, J. (2015). Pensamiento crítico, sujeto y autonomía. México: Centro de Investigaciones y Estudios Superiores en Antropología Social - Cátedra Jorge Alonso.

Velásquez, A. (2006). La Alfabetización Científica y Tecnológica en el proceso de enseñanza - aprendizaje de la Física. Revista Iberoamericana de Educación, vol. 38, 1- 14.

Velásquez, A. (2011). Una mirada crítica a la didáctica de las ciencias, la educación científica y el papel de las TIC en la educación superior. Ibagué: Universidad del Tolima.

Zuleta, E., \& Valencia, A. (1991). Colombia: violencia, democracia y derechos humanos. Bogotá: Altamir Ediciones. 